\title{
Retransmission strategies for cyclic polling over wireless channels in the presence of Interference
}

\author{
Giovanni Gamba, Student Member, IEEE,Federico Tramarin, Student Member, IEEE, \\ and Andreas Willig, Member, IEEE
}

\begin{abstract}
In this paper we consider retransmission strategies for centralized cyclic polling-based systems over wireless channels subject to external interference. The considered strategies differ in the time when retransmissions for one particular node are carried out, and in the number of retransmissions that can be carried out for one node. We show experimentally and by simulation that two related strategies introduced in this paper, called the queueing-based strategies, significantly outperform the traditional strategy (in which all admissible trials towards one node are carried out subsequently) in terms of the average number of nodes that cannot be successfully served in a cycle. These performance gains are achieved without increasing the average total transmission effort.
\end{abstract}

Index Terms-Wireless industrial networks, Cyclic polling, Retransmission strategies

\section{INTRODUCTION}

In many wired industrial networks like for example WorldFIP or PROFIBUS-DP [2] a large share of the overall traffic comes from periodic exchanges of data between a central controller and its attached sensor / actuator stations (which we henceforth call nodes). This traffic typically is allocated to a cyclically repeated periodic window, which is reserved exclusively for the treatment of periodic traffic, whereas sporadic traffic is handled in a separate time window. These two windows alternate in time.

In wireless industrial networks [3], [4] it can be anticipated that the same will be true, but as opposed to wired transmission media, the wireless exchange of data is much more subject to a number of disturbances, including external interference. This interference can affect the successful delivery of packets, and subsequently the controller might have to operate on an inconsistent view of the physical process. Taking the underlying wireless physical layer as given and assuming it is not perfectly reliable, a major control knob to improve the delivery rate are link-layer retransmissions. To accommodate retransmissions for cyclic traffic, extra time has to be allocated in the periodic window. This extra time can be used in different ways. In the most straightforward approach, the bounded immediate retransmission (BIR) approach, for each node there is a limited number of transmission trials available, which are handled successively, i.e. without serving other nodes in between two trials. When all trials have been exhausted

Giovanni Gamba is with the Italian National Council of Research, CNRIEIIT. Federico Tramarin is with the Department of Information Engineering, University of Padova, Italy. Andreas Willig is with the Department of Computer Science and Software Engineering, University of Canterbury, Christchurch, New Zealand.

A preliminary version of this work appeared in [1] and the controller has not received the requested data or acknowledgement frames, the node is regarded as having failed during this cycle. Due to the time-varying nature of errors and interference on the wireless channel it might well happen that a node failing within the current cycle can be successfully served in the next cycle.

In this paper we consider different retransmission strategies for centralized wirelesss industrial systems subjected to external interference. We demonstrate experimentally and by simulation that there are alternative strategies which can reduce the fraction of failed nodes during the periodic window as compared to the BIR strategy. Some of the alternative strategies do not insist on making all trials for a node subsequently, and some allow in a very simple fashion to use the time for trials that "good" nodes have not used in order to serve "bad" nodes with an increased number of trials. We also demonstrate that in scenarios with unbalanced interference (i.e. where some nodes are systematically more prone to interference than others, because they are closer to the interferer) a simple adaptive re-ordering of the polling sequence can significantly increase the performance even for the BIR scheme.

The experiments are carried out with Telos motes platforms carrying ChipCon CC2420 transceivers that are IEEE 802.154-compliant [5], [6] and which operate in the $2.4 \mathrm{GHz}$ ISM band. This band is a license-free band used by several technologies (e.g. Wifi, Bluetooth) and therefore in many environments there is significant interference. The simulations consider a system with a similar physical layer. For both the experimental and the simulation-based assessment it is assumed that the interference is generated by a static interferer (like e.g. a Wifi device) which is not influenced by the controllers or the nodes periodic transmissions (i.e. even if the interferer runs a CSMA-type MAC, it does not recognize the ongoing periodic transmissions).

The paper is structured as follows: in the next Section II we discuss related work. In Section III we explain our assumptions on the network, the organization of a cycle, the operation of the interferer and the major performance measures. Following this, in Section IV we describe the retransmission strategies considered in this paper. In Section $\mathrm{V}$ we explain the simulation setup and present the results of a simulation-based performance study. In Section VI we do the same for the experimental study. Finally, in Section VII we offer our conclusions. 


\section{RELATED WORK}

A number of studies have addressed the problem of interference among different wireless systems. In [7] a theoretical and simulative analysis of interference among WiFi and Bluetooth networks is offered, while in [8] a similar approach is used to evaluate interference among WiFi and IEEE 802.15.4 nodes. A more complex model and an exhaustive set of simulations for the mutual interference between IEEE 802.15.4 and WiFi are presented in [9].

In [10] a fully theoretical and experimental approach is provided in order to evaluate the performance of an industrial polling system based on IEEE 802.15.4-compliant nodes. The cyclic polling is quite similar to the BIR scheme considered in this paper, apart from the CSMA/CA setup. The analyzed interference scenario used a pulsed interference with fixed burst and gap times. The same polling scheme is used in [11]. In this paper the presence of real-life interference (Bluetooth, WiFi, ZigBee interferers) on a IEEE 802.15.4 network is experimentally investigated varying several CSMA/CA parameters. In all these testbeds the standard TinyOS BIR scheme is used, and no investigation on retransmission strategies is offered.

The present work is an extended and revised version of the conference paper [1], where a first set of preliminary results was presented. The present paper extends the conference version amongst others by adding additional polling schemes to the simulations and measurements and by investigating the per-node fairness and uplink-packet interarrival times among the different schemes.

Wireless fair scheduling schemes discussed in [12], [13] exploit the fading nature of the wireless channel to serve different nodes when the channel towards the current node becomes bad (see also [14] for an early facet of this idea). These schemes have some similarities to the queueing-based schemes proposed in this paper, but most of the publications regarding wireless fair queueing concentrate on aggregated throughput (possibly subject to fairness constraints) and do not consider packet deadlines. Furthermore, they often assume independent channels among the involved stations. To the best of our knowledge, the queueing-based retransmission schemes proposed in this paper have not been described so far in the context of industrial communications, nor have they been investigated under external interference, where several channels suffer simultaneously (i.e. are correlated).

\section{SySTEM MODEL AND ASSUMPTIONS}

\section{A. Network model}

We consider a system of one central station (the controller) and a number $N$ of sensor / actuator stations (the nodes). When we do not want to discriminate amongst node and controller, we simply speak of a station.

All stations are stationary. The set of nodes does not change over time and all node addresses are known to the controller. It is assumed that the network has already been set up, i.e. all nodes have successfully associated to the controller.

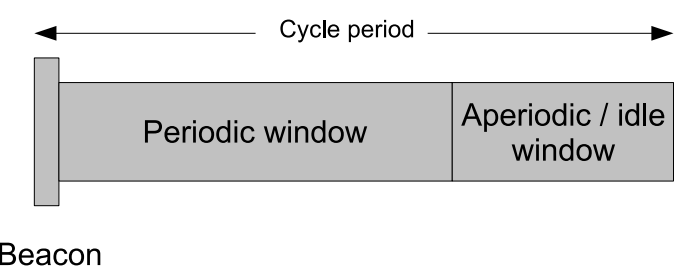

Fig. 1: Organization of transmission cycle

All stations share a common wireless medium (i.e. work in the same frequency band) such that each node can communicate with the controller. On the physical layer we assume that all stations are compliant to the IEEE 802.15.4 standard. They operate in the $2.4 \mathrm{GHz}$ ISM band and use all the same modulation scheme and transmit power. The controller is considered as a master and the nodes are considered as slaves. A polling-based scheme is adopted, i.e. a scheme in which the controller sends a request-frame to an individual node (possibly carrying some output data for this node) and the node immediately answers with a response-frame (again, possibly carrying some data). The controller is able to determine whether it successfully received a response-frame or not, i.e. it can obtain binary feedback. It is assumed that a client-server interaction pattern is adopted, i.e. the data generated by one node is not immediately relevant to any other node, but only to the controller.

An important assumption is that the controller does not perform any carrier-sensing operation before transmitting a request-frame, and vice versa a node does not perform carriersensing before transmitting a response packet. This is commonly satisfied in industrial master-slave systems, but has the disadvantage that the system has no chance to respond directly to external interference.

\section{B. Organization of the cycle}

The cycle organization is fairly straightforward and shown in Figure 1. A cycle has a fixed duration (cycle period) of $\Delta_{c}$ seconds. A cycle starts with a synchronization or beacon packet broadcast by the controller to all nodes. The main purpose of this packet is for the nodes to maintain time synchronization with the controller. It is not strictly necessary for a node to receive each beacon and in this paper we do not care about the beacons anymore.

The remaining cycle is sub-divided into two windows. In the first window, the periodic window, the controller handles all periodic traffic. The size of the periodic window, $\Delta_{p}$, is chosen so that each node can be polled at least once (involving transmission of a request packet and a response packet) and furthermore some additional time budget is available to perform retransmissions (which again consist of request and response). All these retransmissions, however, must take place within the periodic window. It may happen that for one or more nodes the controller has not obtained any response at all at the end of the periodic window. In this case, the nodes are said to have failed during the cycle. In practical implementations, nodes failing successively for a number of periodic windows would be removed from the polling sequence. We do not adopt 


\begin{tabular}{llll}
\hline Burst k-1 & & Burst k \\
\hline & Gap k+1 & Burst k+1 \\
\hline
\end{tabular}

Fig. 2: Interference pattern

this policy in this paper, i.e. nodes are always polled, no matter how often they have failed in the past.

The following aperiodic/idle window is of no further concern to us - it can (like e.g. in WorldFIP) be used to carry out aperiodic message exchanges, or it can be a pre-defined idle phase which the controller can use to process the responses. The only relevant assumption about this window is that it is in no way available for handling periodic traffic anymore.

\section{Traffic model}

For the purposes of this paper it suffices to assume a very simple traffic model: in each cycle the controller produces for node $k$ output data of length $l_{o, k}$ and vice versa node $k$ produces input data for the controller of length $l_{i, k}$. In addition, a fixed-length packet header and trailer consisting of $l_{h}$ bytes is added to the user data. We make the simplifying assumption that all output data have the same length, i.e. $l_{o, k}=l_{o}$ for some constant $l_{o}>0$ and all $k$. Similarly, for the input data we assume $l_{i, k}=l_{i}>0$.

We assume that there is a total number of $N \times K$ trials available ( $K>1$ and $K$ being an integer) during the periodic window.

\section{Interference model}

In both our experiments and the simulations the interference is generated artificially from a relatively simple stochastic process. We furthermore assume that external interference is the dominating source of channel errors, other sources like fading have not been considered. The interferer does not perform any carrier-sensing on the common channel, so that the interferers behaviour is not influenced by the controller and the nodes.

The interference process is depicted in Figure 2. It alternates between bursts, during which the external interferer transmits, and gaps, where the external interferer is silent. The interferer is static and uses the same transmit power in all the bursts. It is assumed that the interferer uses a completely different rate and modulation scheme than our IEEE 802.15.4 stations, so that the interferers signals can be regarded as white noise for the controller and nodes. Therefore, the interferers activities correspond to a time-varying noise level. This is common assumption for modeling external interference.

To keep the generation of the interference simple, the gap lengths are modeled as an independent and identical distributed (IID) sequence of exponential random variables of a given average gap length. The burst lengths are iid and have a uniform distribution drawn from a given interval $\left[b_{l}, b_{u}\right]$. This choice reflects maximum uncertainty about the length of the interference bursts.

There is, however, a crucial difference between our experiments and the simulations regarding the directivity of the interferer. In the experiments we have used a directional antenna with a relatively narrow beam. If we point this antenna to one node, then the reception of this node is distorted, but the transmissions of this node can still be heard by the other nodes when they are outside the antenna beam. In contrast, in the simulations the interferer is assumed to have a perfectly omni-directional antenna. For this reason the experimental and simulation results are not directly comparable.

\section{E. Major performance measures}

The main performance criterion is the delivery rate. More specifically, the downlink delivery rate gives the average fraction of nodes which successfully receive their output data within the periodic window. Vice versa, the uplink delivery rate gives the average fraction of nodes which successfully deliver their data to the controller within the periodic window. Since controller and nodes use a request-response communication pattern, the downlink delivery rate is always at least as large as the uplink delivery rate. We therefore concentrate on the uplink delivery rate, or equivalently, the (average) number of nodes for which no uplink packet is received (which we denote as average number of unserved nodes). A related measure is the cycle loss, which for an individual node denotes the percentage of cycles where the controller does not successfuly receive an uplink packet.

A second important criterion is the fairness index of each scheme. For a specific setup with $N$ nodes, we compute at the controller for each node the average time between arrivals of responses, and the fairness index is defined as the difference between the maximum and minimum of these averages (taken over all nodes). Please note that the fairness index is expressed in seconds, smaller values indicate better fairness.

\section{CONSIDERED RETRANSMISSION STRATEGIES}

We describe the retransmission strategies considered in this paper.

\section{A. Bounded Immediate retransmissions (BIR)}

This is a baseline scheme mimicking the atomic behavior of packet transactions in PROFIBUS. It works as follows: for each node at most $K$ trials can be made. If the first trial fails, the controller immediately starts the next trial, until either all $K$ trials have been exhausted, the controller successfully receives a response, or the periodic window ends. When processing for node $i$ has ended and there is still time available, processing for the next node $j$ starts. Node $j$ is not served in between two trials for node $i$.

Technically, this scheme is a simple ARQ scheme with a bounded number of immediate retransmissions. The efficiency of this scheme (and all other schemes as well) over fading channels (or other types of non-stationary channels like the interference channels considered in this paper) will depend on the channel coherence time, i.e. the time for which the channel does not change its characteristics [15]. When this time is large enough to cover several packets in succession, then a packet transmitted to a node with a currently bad channel will likely fail. The BIR scheme furthermore has the disadvantage that the trials not used by one node cannot be used by another node. 


\section{B. Unbounded Immediate retransmissions (UIR)}

This scheme is similar to the BIR scheme in that all trials for node $i$ are carried out in succession without serving other nodes in between, but in the UIR scheme there is no limit on the allowed number of trials - the controller can perform as many retransmissions for a node as would fit into the periodic window. This scheme, which is of little practical value, has been included as a baseline scheme to stress the effect of bounding the number of retransmissions. One obvious problem with this scheme is that when one of the stations polled early in the cycle suffers from a bad channel, the controller will spend a lot of time with this station at the cost of not treating the remaining stations.

\section{Queued retransmissions $(Q R)$}

The controller maintains a FIFO queue of node addresses. When this queue is empty or the periodic window is exhausted, the controller stops working on periodic data exchanges. Otherwise, the controller removes the first entry, say address $i$, from the front of the queue and performs one single trial towards node $i$. If this trial fails, a new entry for $i$ is appended to the tail of the queue and the next head-of-line entry is served. At the beginning of the periodic window the queue is initialized with all $N$ addresses in the sequence from 1 to $N$.

In this approach first all $N$ nodes are tried once. The successful nodes are not considered any further during the current cycle, and for the failing nodes a new trial is appended to the queue. This has two effects. First, the spacing between the first trial and the first retransmission is larger on average than with immediate retransmissions. This allows to deal with larger channel coherence times. The spacing between the second and third trial is in general smaller and random. Secondly, the number of retransmissions that can be performed for one node might be larger than $K$, provided other nodes have required fewer than $K$ trials. A further benefit of this approach is its simple implementation.

\section{Adaptive versions (AQR, AUIR, ABIR)}

In the previous three strategies $(\mathrm{QR}, \mathrm{BIR}, \mathrm{UIR})$ the sequence in which nodes are initially polled is fixed from $1,2 \ldots N$.

In our adaptive schemes the controller maintains for each node long-term statistics about the relative frequency with which one trial is successful. At the start of a cycle, instead of initializing the polling sequence with $1,2, \ldots, N$, it is initialized according to the average success probability for a trial: nodes having the largest success probability (and hence requiring the fewest average number of trials until success) are tried first. By picking the nodes with the smallest average numbers of trials first, the average number of nodes that can be successfully served within the periodic window is maximized.

The adaptive schemes are intended to be implemented in devices with reduced computational and memory capabilities, so the estimation complexity as well as memory usage should be limited. Therefore we have adopted a simple exponentially weighted moving average estimator, that is, in other words, a first order infinite impulse response (IIR) filter. The estimator of the trial success probability after trial $n+1$ is:

$$
\bar{s}_{n+1}=\alpha \bar{s}_{n}+(1-\alpha) s_{n+1}
$$

where $\bar{s}_{n}$ is the estimated probability after $n$ trials, $s_{n+1}$ is the outcome (success or failure, represented as 1 and 0 , respectively) of the $n+1$-th trial and $\alpha$ is a parameter. The estimator update for each node is performed with a product and two sums, and requires one memory position to store $\bar{s}_{n}$, the previous step estimation. In the practical implementation a fixed point arithmetic is used, so a normalization and truncation of all the entries in equation (1) are performed. The motivation for choosing this type of estimator is that it "forgets" the past after some time, which is appropriate for time-varying wireless channels. The factor $\alpha$ controls how quick the memory vanishes. Throughout this paper we use $\alpha=0.9$, i.e. most weight is put on the history and new observations influence the estimation only with a weight of $10 \%$. We have experimented with other values of $\alpha$, but we found that $\alpha=0.9$ gave a good compromise between stability and agility of the estimator.

The frequency by which new observations are taken for one channel does in general not allow to track quick channel variations, like e.g. generated by fast fading. However, our results show that already this simple estimator can yield very good gains for static scenarios in which the nodes suffer from interference by different degrees.

\section{Simulation-Based evaluation}

In this section we describe the results of a simulation-based performance study. As already mentioned in Section III-D, the interference model supported by the simulator (with an omnidirectional interferer) differs from the interference created in the measurement setup, which is directional.

We first describe the simulation model, then we present and discuss the results.

\section{A. Simulation model}

The simulation model is using a discrete-event simulator written by the authors in Common Lisp [16]. This simulator includes the protocols used in this paper, a separate interferer node and a wireless channel model linking all stations (including the interferer) together. This channel model supports variable transmit powers, the log-distance path-loss model [15], freely adjustable noise powers and receiver sensitivities. For the wireless channel we assume a narrowband channel following the log-distance path loss model [15], using a reference distance of $1 \mathrm{~m}$ and a path loss exponent of $\gamma=3$. No fading model is used.

The simulation at each packet receiver accurately tracks the SNR level over time. The SNR observed by a single receiver can change due to the generated interference. Within a single packet different interference levels and therefore different SNR levels can be observed. From the SNR level within one part of the packet a bit error (BER) rate is computed, and the number of erroneous bits in this part is drawn randomly from the packet segment length and the computed BER. When the 


\begin{tabular}{|l|l|}
\hline PHY parameters & Value \\
\hline Transmit power $P_{t}$ & $1 \mathrm{~mW}(0 \mathrm{dBm})$ \\
\hline Noise power $N_{0}$ & $-143.0 \mathrm{dBm} / \mathrm{Hz}$ \\
\hline Reference distance $d_{0}$ & $1 \mathrm{~m}$ \\
\hline Reference path loss $\mathrm{PL}_{0}$ & $-20 \mathrm{dBm}(\mathrm{see}[17],[18])$ \\
\hline Path loss exponent $\gamma$ & 3 \\
\hline Modulation and data rate & BPSK @ 250 kbps \\
\hline Transceiver turnover times & 2232 bit times \\
\hline \hline MAC-Parameters & Value \\
\hline Fixed overhead size & 152 bits \\
\hline Beacon payload size & 48 bits \\
\hline Downlink payload size & 48 bits \\
\hline Uplink payload size & $168 \mathrm{bits}$ \\
\hline Max. trials for BIR scheme & 2 \\
\hline Cycle time & $400 \mathrm{~ms}$ \\
\hline Periodic window size & $330 \mathrm{~ms}$ \\
\hline \hline Simulation precision parameters & Value \\
\hline Simulation duration & $86400 \mathrm{~s}$ \\
\hline
\end{tabular}

TABLE I: Fixed simulation parameters

packet contains one or more bit error, it is dropped. A polling trial fails if either a poll request or a poll response is lost. A beacon loss does not influence the cycle, being only a synchronization frame.

The fixed simulation parameters are given in Table I.

The IEEE 802.15.4 standard defines several modulation types. In the $2.4 \mathrm{GHz}$ band the admitted one is an OQPSK. However, the BER expression given for O-QPSK in [6, App. $\mathrm{E}]$ is numerically complex and requires significant simulation times. We therefore have decided to use a less computational intensive BER approximation. As reported in Table I we have chosen a BPSK modulation. While BPSK does not achieve the same BER vs. SNR performance as OQPSK, the results obtained with OQPSK would be very similar to the ones obtained with BPSK, only at slightly shifted interferer transmit powers.

The cycle time has been set to $400 \mathrm{~ms}$. For one single trial (consisting of request and response) the parameters for the packet lengths and the transceiver turnaround times have been chosen so that one trial takes $20 \mathrm{~ms}$. Figure 3 depicts a typical poll cycle: the poll request has a length of 200 bits, whereas the response from the node is 320 bits long. Summing up these quantities and considering two transceiver turnover times we obtain a total of 4984 bits, that correspond to about $20 \mathrm{~ms}$ at the maximum rate for the IEEE 802.15.4 standard. This choice has been made to be consistent with the measurement setup, the turnaround times in the simulation model accommodate the processing delays observed in our setup. The periodic window has a length of $330 \mathrm{~ms}$, which is sufficient to accommodate 16 trials. The overall setup consists of the controller, eight nodes and an interfering node. With eight nodes to serve and 16 trials available we have $K=2$. The controller has been placed in the center (position $(0,0)$ ), the nodes have been placed equidistantly on a circle of $7 \mathrm{~m}$ radius around the center. The rightmost node is placed at position $(7,0)$, the leftmost node at position $(-7,0)$. For the position of the interferer we have considered two settings:

- In the first setting the interferer is placed at position $(7.1,0)$ and therefore disturbs the rightmost nodes most. We call this, somewhat imprecisely, the one-disturbed-

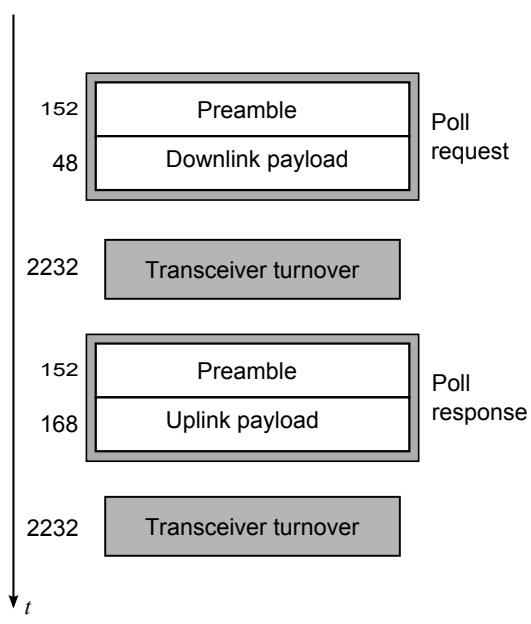

Fig. 3: Data exchange in a typical poll cycle. Numbers represent bits. Data rate is $250 \mathrm{~Kb} / \mathrm{s}$. The total number of bits in a poll cycle is 5032, resulting in about $20 \mathrm{~ms}$ for a cycle.

\begin{tabular}{|l|l|}
\hline IF parameter & Value \\
\hline Transmit power & $\{-20,-30,-50,-52, \ldots,-60\} \mathrm{dBm}$ \\
\hline Avg. gap length & $\{10,30,50\} \mathrm{ms}$ \\
\hline Burst length distr. & $\{U[1,10], U[2,20]\} \mathrm{ms}$ \\
\hline \hline
\end{tabular}

TABLE II: Varied interferer parameters. $U[x, y]$ refers to the continuous uniform random distribution over the interval $[x, y]$.

node scenario. It represents a scenario in which the nodes of a network suffer from interference differently, i.e. some nodes are more disturbed than others.

- In the second setting the interferer is placed very close to the controller, so that all nodes are disturbed in the same way by the interferer and the controller is disturbed most. We call this the all-disturbed-nodes scenario.

In the one-disturbed-node scenario the chosen node positions causes the rightmost nodes to be most affected by interference, while the leftmost node is the least affected. Therefore, for a given interferer transmit power the nodes experience different SNR values. For the presentation of our results we therefore use the interferer transmit power to compare all nodes on the same grounds. After reaching an interference level able to impair all the network, a further increase in the interference power is not expected to modify the performance of the system. The interferer follows the pattern described in Section III-D. For the simulations, we have varied the interferer parameters as summarized in Table II, simulating a highly interfered scenario. A real wireless industrial network would never intentionally operate in such an hostile environment, but the analysis is intended as a worst case perspective showing the effects of unpredicted interferers.

\section{B. Results}

All our results for the all-nodes-disturbed scenario are very similar to each other and are not presented in detail here: the BIR and ABIR overlap almost completely over the whole 
range of interferer transmit powers, the other four schemes overlap over the the largest part of the range, and the (A)BIR schemes show worse performance in terms of the average number of failed nodes than the other schemes. Since all nodes suffer in the same way from interference, the adaptive schemes make no difference over their non-adaptive counterparts. The performance disadvantage of the (A)BIR schemes can be explained by the lack of "trial re-use", i.e. each node is assigned the same maximum number of attempts to fulfill the polling, irrespective of the actual needs.

The one-disturbed-node scenario reveals, instead, a more variable behavior and hence this section is focused on it. Figures 4 and 5 show the average number of unserved nodes for the short and long average interference burst lengths respectively and for an average interference gap length of 10 ms. In Figure 6 we show similar results for the case of $30 \mathrm{~ms}$ average gap lengths and short bursts. The results for the 50 $\mathrm{ms}$ case look very similar to the curves for the $30 \mathrm{~ms}$ case and are not shown here.

In all these figures, a "transient" zone is noticeable in the leftmost part of the graphs. For all schemes there is significant performance variation for interferer transmit powers between -60 and $-50 \mathrm{dBm}$, while for interferer transmit powers larger than $-50 \mathrm{dBm}$ the performance of each scheme does not change much anymore. This is due to the increasing number of nodes impaired by the interferer. For values of interference greater than $-50 \mathrm{dBm}$ all the nodes are involved and hence the number of unserved nodes saturates.

For the $10 \mathrm{~ms}$ average gap duration case and short interference bursts, it can be seen in Figure 4 that for the shorter burst lengths the BIR and ABIR schemes show consistently the worst performance due to the lack of trial re-use. The UIR scheme performs better since it allows as many attempts as necessary to leap over the (short) interference burst. The performance of UIR degrades as the interference bursts become longer, as is exemplified in Figure 5 for the case of long interference bursts and $10 \mathrm{~ms}$ average gap duration. Here, UIR has the worst performance (followed by the BIR and ABIR schemes) since for long interference bursts UIRs persistence on serving a bad node has consequences for all following nodes, the last ones possibly not being polled at all.

The AQR and QR schemes show very similar behaviors. AQR does not significantly improve the basic QR scheme since the queuing mechanism is already a "self adapting" policy that postpones the impaired nodes to be served after the good ones and the only difference between the two schemes is the starting order, that is scrambled just after few steps. The AUIR scheme shows the best performance especially in the "transient" region. Apart from BIR and ABIR, all the other schemes perform similarly; this proves further the effects of an unbounded number of retransmission trials.

It is interesting to note that the relative performance of the UIR and (A)BIR schemes depends on the length of the interference bursts. The difference between the UIR and the QR scheme points to the value of increasing the spacing between the first trial and the first retransmission. It is also very interesting to note the significant improvement in performance of the AUIR scheme as compared to the UIR scheme. It should also be mentioned that the performance of all schemes depends, for given interference gap length, of course on the average interference burst lengths, so that longer bursts lead to overall reduced performance. This can be seen from comparing the ranges on the y-axis of Figs. 4 and 5.

For $30 \mathrm{~ms}$ and $50 \mathrm{~ms}$ average gap times (see Figure 6) and short interference burst lengths the BIR and ABIR schemes show the worst performance, whereas the other schemes do not differ very much. This figure highlights the effect, already stressed in Figure 4, of bounding the number of retransmissions for each node. In fact, two distinct set of curves are noticeable. The same is true for the longer bursts at $30 \mathrm{~ms}$ average gap duration and also for long and short burst lengths at $50 \mathrm{~ms}$ average gap duration.

As a conclusion, when all nodes are distorted in the same way (as in the all-nodes-disturbed scenario), there are practically no differences between UIR, AUIR, QR and AQR, but all these schemes are significantly better than BIR and ABIR. When the interference situation among the nodes becomes heterogeneous, the performance of the schemes starts to differentiate as well and the AUIR scheme shows the best performance, followed by $\mathrm{AQR}$ and QR. On the other hand, the performance of the BIR and ABIR schemes is either the worst one or the second-worst one, so they should be avoided in practice. The likely explanation for this is (A)BIRs inability to use unused trials from good nodes to increase the number of trials for nodes with worse interference conditions.

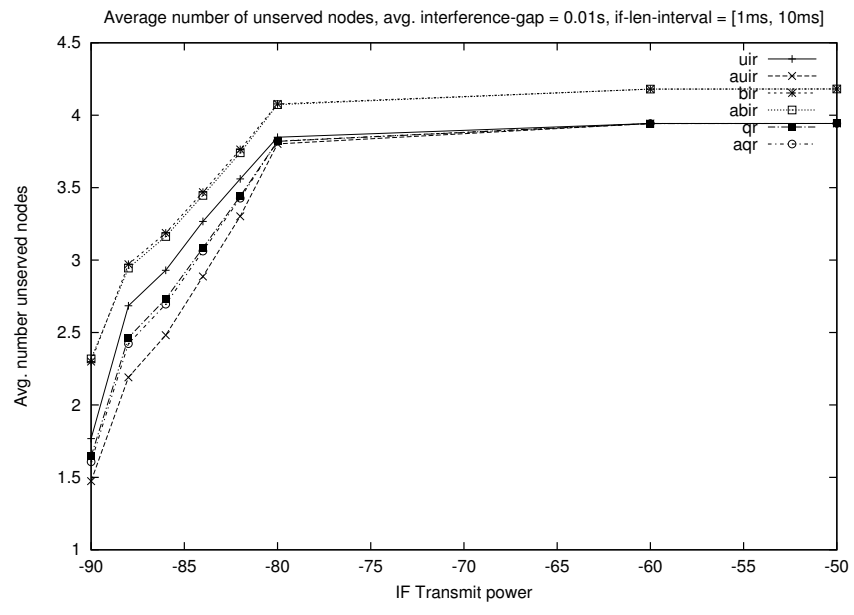

Fig. 4: Average number of unserved nodes in the onedisturbed-node scenario, short interference bursts, average $\mathrm{IF}$ gap time $=10 \mathrm{~ms}$

We now look at the fairness indices of the different schemes, which we show for long interference bursts and an interference gap time of $10 \mathrm{~ms}$ in Figure 7. Taking aside the UIR scheme (which for interferer transmit powers between -60 and -50 $\mathrm{dBm}$ assumes extremely high values, not displayed properly), it can be seen that the AQR scheme has the best fairness, followed by the ABIR and, for all but the highest interferer transmit powers, the QR scheme. The AUIR scheme, which showed the best performance in terms of the number of unserved nodes, now shows the worst performance in the inter- 


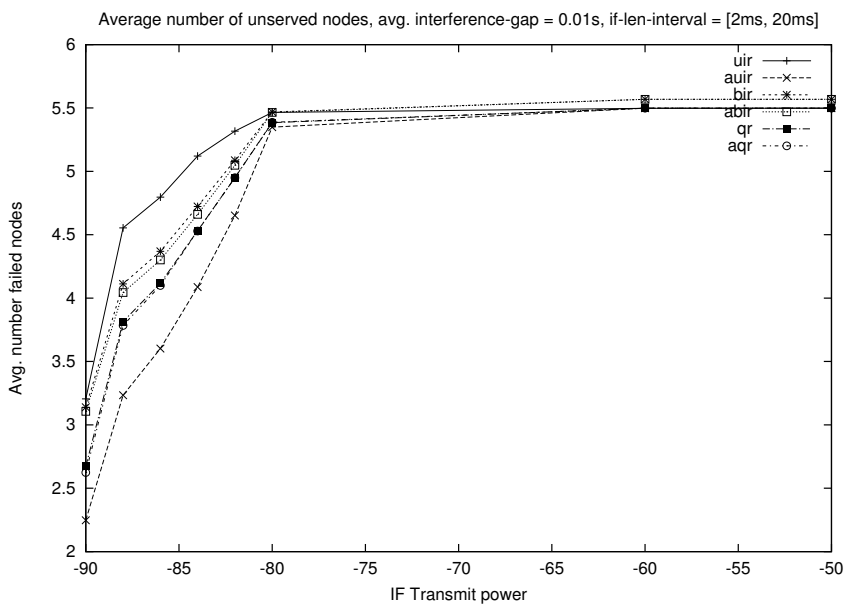

Fig. 5: Average number of unserved nodes in the onedisturbed-node scenario, long interference bursts, average IF gap time $=10 \mathrm{~ms}$

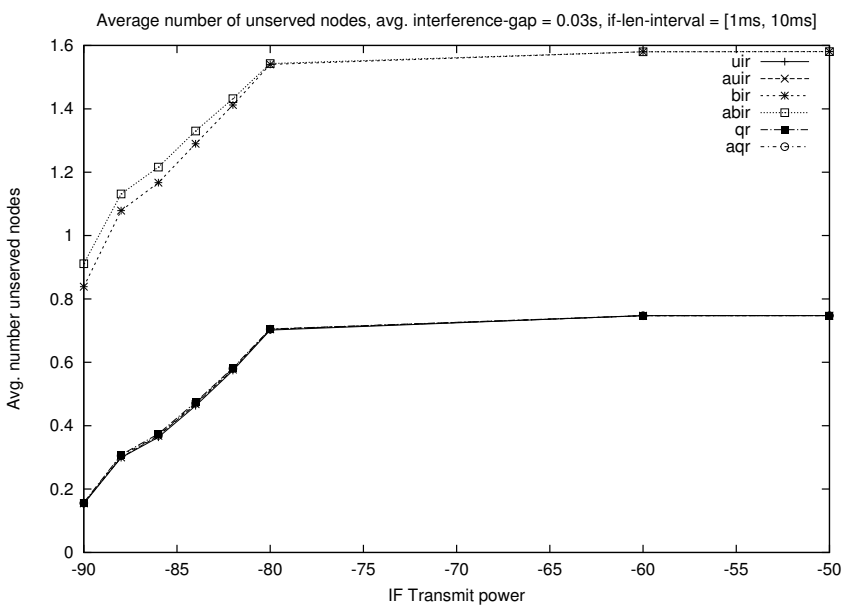

Fig. 6: Average number of unserved nodes in the onedisturbed-node scenario, short interference bursts, average IF gap time $=30 \mathrm{~ms}$

esting range between -60 and $-50 \mathrm{dBm}$, and never approaches the fairness performance of $\mathrm{AQR}$ and $\mathrm{ABIR}$. The transitional behaviour (the "bump") of all adaptive schemes between $60 \mathrm{~dB}$ and $-50 \mathrm{dBm}$ can be explained from fluctuations in the adaptive packet loss rate estimator (compare Equation 1): for large enough interferer transmit powers the interferers transmissions "reliably" destroy transmitted packets, therefore the estimator directly observes the interferer statistics. In the range with low interferer transmit powers (between -50 and $-60 \mathrm{dBm})$ not all packets are destroyed by the interferer, and therefore the observations of the interferers behaviour become themselves noisy.

\section{Vi. Measurement Results}

\section{A. Measurement setup}

Figure 8 shows the measurement setups that have been used in our experiments. The sensor network comprises of one

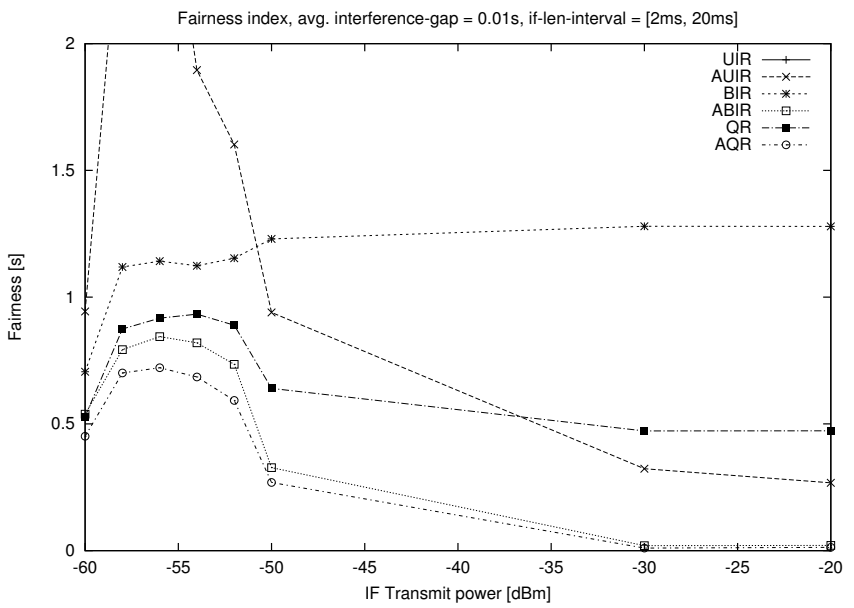

Fig. 7: Fairness indices in the one-disturbed-node scenario, long interference bursts, average IF gap time $=10 \mathrm{~ms}$

controller and $N=8$ nodes. All the experimental parts of this work have been performed in a non-anechoic room, so nonideal effects of a real-life environment could not be excluded. However a preliminary scan of the chosen band showed no spurious emissions.

For our experiments we have adopted the one-disturbednode and all-disturbed-nodes scenarios described in Section V-A. In our all-disturbed-nodes setup the nodes have been evenly spaced on a semi-circumference of $60 \mathrm{~cm}$ radius, with the controller in the center. A directional antenna has been placed far away from nodes in order to satisfy the far-field hypothesis. We verified that the received signal strength at each node is approximately the same. This allow us to assume that, in the area of sensors, the electromagnetic field is isotropic. In our one-disturbed-node setup the interference has been directed only toward one node, namely node 3 .

We jointly show both setups in Figure 8. Two antennas have been sketched, a switch plugs the RF interference signal into the left antenna in the one-disturbed-node setup, with the beam directed only toward the node close to the antenna. The interference power has been regulated to disturb only communications regarding this node.

We used the TinyOS 2.1 operating system to develop the application of each node. This application implements the overall polling scheme (beacon transmission, poll-request and poll-response generation on controller and nodes, respectively) and four of the different retransmission strategies used in this work, namely UIR, BIR, QR and AQR. The underlying protocol stack is basically the default protocol stack delivered with TinyOS 2.1, but we modified the CSMA/CA and CCA mechanisms to effectively get rid of the carrier sense functionalities.

The interference signal behaviour has been described in Section III-D. The real signal used in our experiments was an AWGN signal, with a bandwidth of $5 \mathrm{MHz}$, centered over the same IEEE 802.15.4 channel used for the WSN (in this case the 26-th, i.e. $2.48 \mathrm{GHz}$ ), produced by a RF Agilent E4433B signal generator. To generate the described pattern we have 


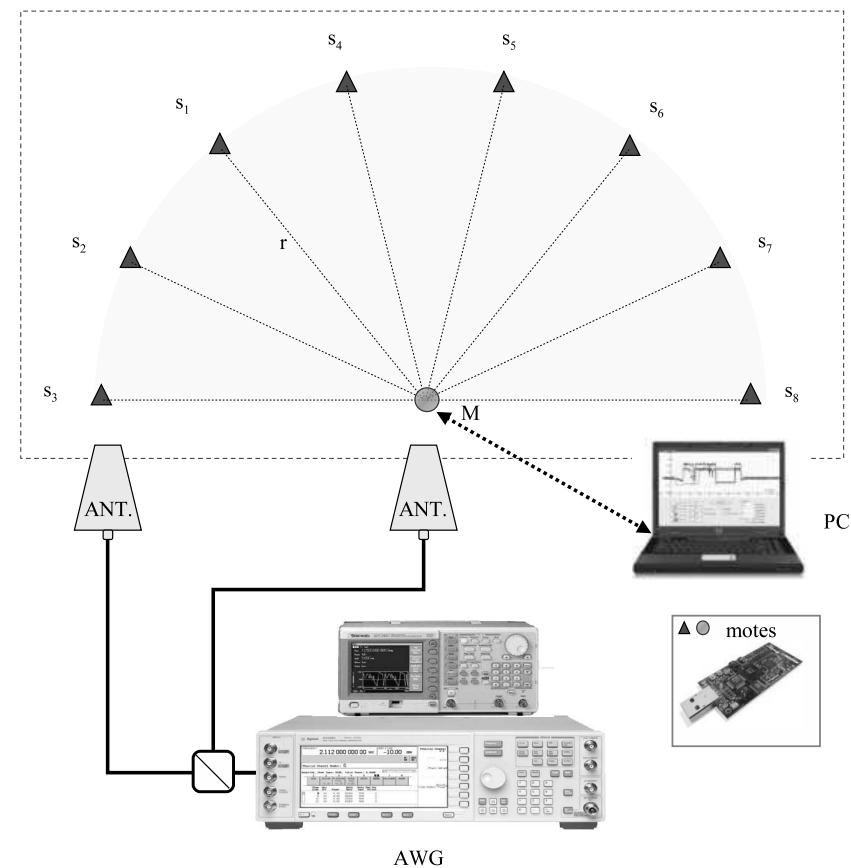

Fig. 8: Measurement setups used in experiments.

used a pulsed mode, using a baseband signal generator as trigger. The baseband signal generator is able to reproduce an arbitrary waveform from a succession of values from a file. Thus we have first generated this succession of points sampling the stochastic process described in Section III-D, then we have used the baseband signal generator to reproduce a signal according to the succession. Finally, we have used this signal as a trigger for the RF signal generator, to switch on and off the radio.

Note that in this approach the interferer does not react (by not using any carrier sense mechanism) to the traffic generated by the sensor network.

The RF signal produced has been irradiated with a directional antenna placed behind the controller, with the main lobe covering the WSN area.

The different level of SNR in the uplink and downlink experienced in [1], has been fixed increasing the distance of antenna from the network and using the minimum allowed $(-25 \mathrm{dBm})$ transmission power. In fact, in the experiments we carried out in [1] we used the IF to block only the reception of the poll response by the controller, setting an SNR of $0 \mathrm{~dB}$ in the up-link transmission. Now, in the all-disturbednodes scenario an interferer can now prevent both uplink and downlink transmission, instead.

\section{B. Results}

In our experiments we have fixed some parameters with respect to the simulation, in order to shorten the time requirements for the tests. First, we have fixed the IF power, which was set to $8 \mathrm{dBm}$ at the instrument side (RF signal generator). With this setting we achieve a SNR $<0 \mathrm{~dB}$ both at the controller and at the nodes. The channel has been chosen as channel 26 of the standard, centered at $2.48 \mathrm{GHz}$. This

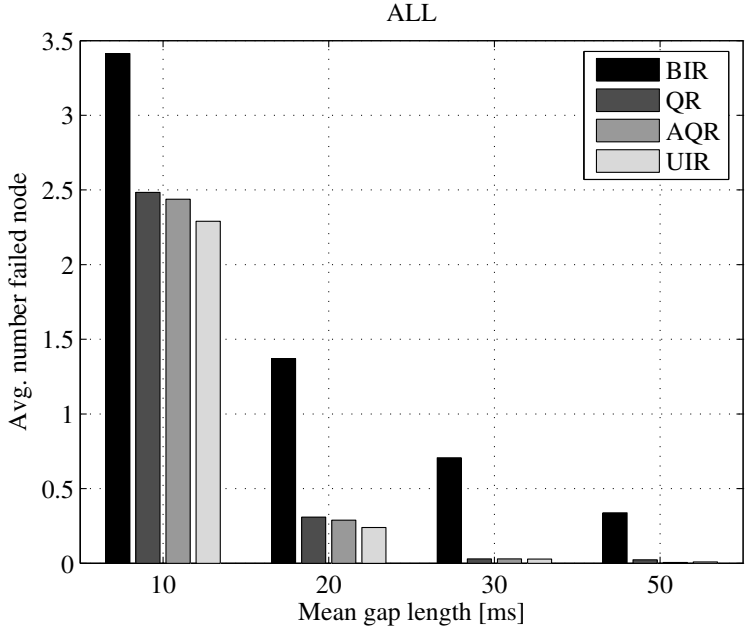

Fig. 9: Average number of unserved nodes in the alldisturbed-node scenario, $U(1,10) \mathrm{ms}$ interference bursts, average IF gap time on the $\mathrm{x}$-axis

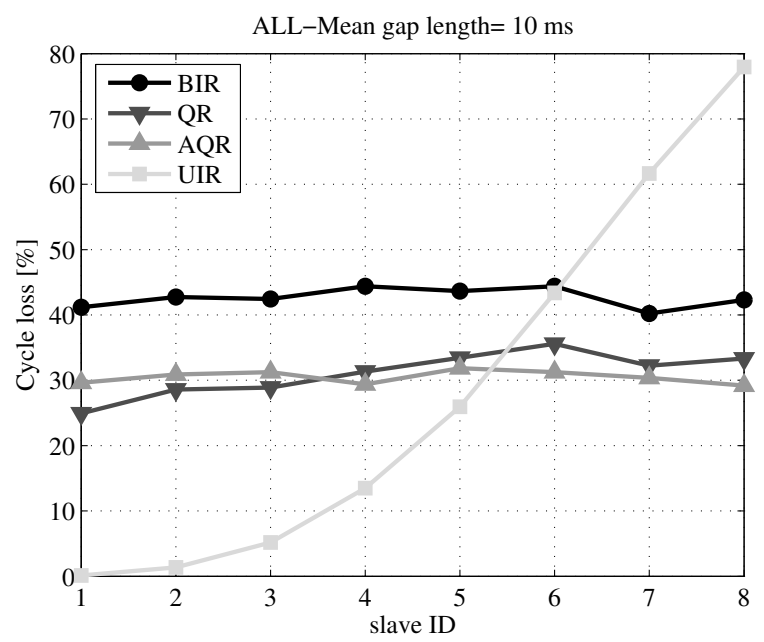

Fig. 10: Cycle loss for all-disturbed-node scenario, $U(1,10)$ ms interference bursts, $10 \mathrm{~ms}$ average IF gap time $v \mathrm{~s}$ node ID.

is the highest available IEEE 802.15.4 channel, which does not overlap with the commonly chosen Wifi channels at our department.

The MAC parameters have been chosen according to Table I. The IF signal has been reproduced with the same characteristics as presented in Section III-D. We have considered four different mean values for the exponential random variable (representing gap spaces between bursts), namely 10, 20, 30 and $50 \mathrm{~ms}$. The burst lengths are randomly chosen from $U[1,10] \mathrm{ms}$, which corresponds to the short interference burst lengths considered in Section V-A.

In order to obtain comprehensive statistics from the data collected during the measurements, we have chosen to run a single experiment for approximately 15 minutes, allowing the transmission of $\mathrm{M}=2300$ cycles. Each experiment has been carried out with three repetitions, spaced in time, to avoid 
correlations with environmental parameter variations. In all cases the results of the repetitions were very close to each other, so the first repetition has been used for statistics and plots.

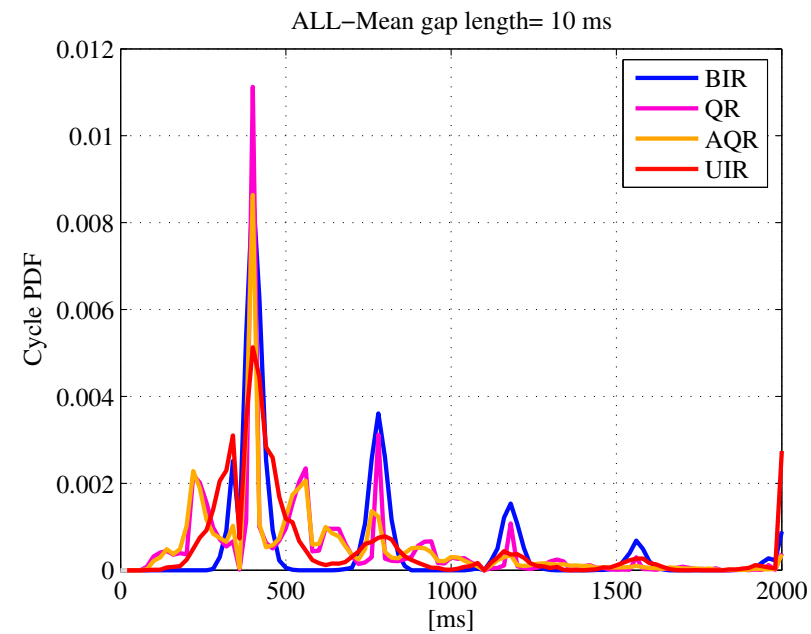

Fig. 11: Measured uplink cycle iat probability density function: average over the nodes.

The results regarding the one-disturbed-node scenario are very close to the ones obtained in [1] and hence are not reported here. In the following only the all-disturbed-nodes scenario results are shown.

Figure 9 shows the average number of unserved nodes for the different retransmission schemes. Please note that this is a mean value taken over all the nodes and is not useful to stress inequalities and unfairness in the performance of different nodes. Figure 10 specifically underlines such a perspective instead. It depicts different cycle loss experienced by each node using different retransmission schemes. The following points are noteworthy:

- In terms of the average number of unserved nodes (Figure 9) the BIR scheme is the worst approach, since it allows only $K$ transmission attempts to each node without a dynamic allocation of trial budget. The AQR scheme is slightly better than the QR scheme and UIR performs as the best one. As expected, longer interference gap space allows better performance.

- A more in-depth analysis (Figure 10) shows that UIR is not a fair approach because the nodes experience very different cycle loss: node number 8 reaches $80 \%$ of cycle loss, and in general the cycle loss increases with the node index in our setup. This behavior can be explained by the fact that this strategy spends an unbounded number of trials for each node to get a successful transmission, therefore last nodes will have less trials to carry out their data delivery. In other words, this means that devices with high node ID would seldom, if ever, be correctly polled.

- The results reported are obtained with the IF average gap value of $10 \mathrm{~ms}$ and hence in a highly interfered scenario. Less aggressive (i.e. with IF average gap value of 20, 30 and $50 \mathrm{~ms}$, as can be seen in Figure 9) interference patterns show similar results even if difference among polling policies are less sharp.

In a polling system it is often important to guarantee that inter-arrival times between uplink (and downlink) packets are fixed or at least have low variance. To this aim in the following for each node the uplink inter-arrival time (iat) is analyzed through a normalized histogram, also referred to as probability density function (PDF). Our histograms reflect the raw difference between timestamps of uplink packets, taken immediately after their arrival. The main lobe of the pdfs is around $400 \mathrm{~ms}$ (the cycle time), but if some cycle is lost, the pdfs show spikes around multiples of $400 \mathrm{~ms}$. Figure 11 shows the pdfs for the different retransmission schemes. In these pdfs the samples for all nodes have been combined. As for Figure 9 this averaged graph can not appreciate the fairness of the different polling policies. Figure 12 compares the same pdfs, showing the behavior of each node. It is worth noting that:

- Figure 11 shows that BIR scheme is the most "deterministic" one in the sense that the spikes around the multiples of $400 \mathrm{~ms}$ are very sharp. The high cycle loss of BIR is reflected in the number and amplitude of the side lobes. $\mathrm{QR}$ and $\mathrm{AQR}$ are very similar to each other. They follow the spikes of BIR, but have more probability mass in between these spikes. AQR's lower cycle loss causes its secondary spikes to be smaller than those of QR. UIR has a very wide main lobe, but seems not to have much probability mass in secondary lobes .

- Figure 12 underlines differences in a per-node fashion. Figure 12(a) shows for the BIR scheme that for increasing node IDs the pdfs become less tight around the multiples of 400 ms: peaks are getting lower and wider. Figure 12(b) shows a huge unfairness for the UIR scheme: node 1 is quite deterministic and loses no packets, while node 8 has many secondary wide lobes. Figure 12(c) shows for the QR scheme that most variation occurs around the main lobe. The symmetric peaks around the main lobe shift and get lower increasing node ID. Figure 12(d) shows that AQR policy is the most fair: the pdfs of the different nodes are almost identical.

In a polling system it is also of interest to describe the maximum delay experienced using a particular polling scheme. To this extent the maximum delay for each baseline scheme is analyzed through a bar plot. This interesting quantity has been calculated resuming data on inter-arrival times, and considering only those polling cycles leading to a successful transmission of data to the master node. The maximum delay has been defined as the maximum inter-polling time between those polling cycles for each node. Figure 13 shows this plot for each average gap length. The bar plot highlight again that the BIR scheme has the most deterministic behavior, in the sense that it defines an upper bound for the delivering delay of a packet unrelated from the maximum polling window time. Conversely, QR and AQR schemes, while do not change their performances with the gap length increase, saturate the polling window time with their transmissions. This expense, however, leads to an higher delivery rate for these two polling scheme. Furthermore, UIR scheme seems to increase its performances 


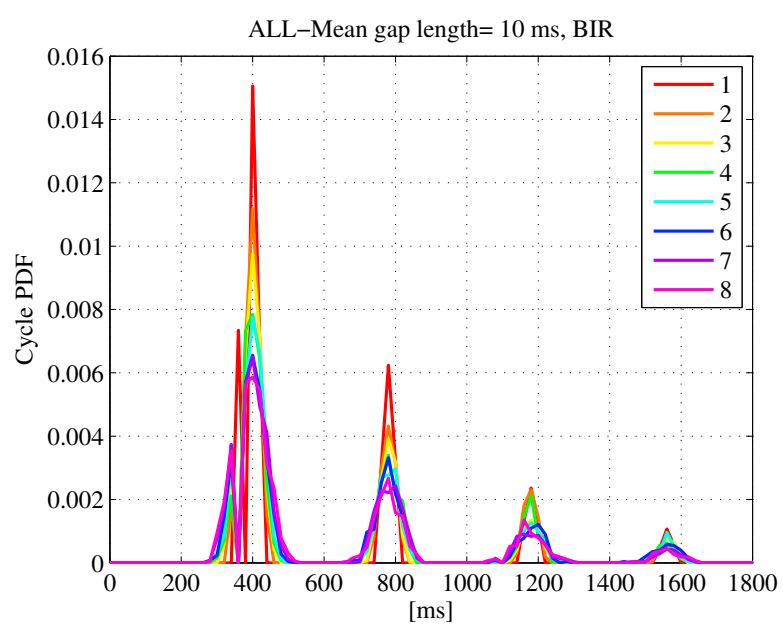

(a)

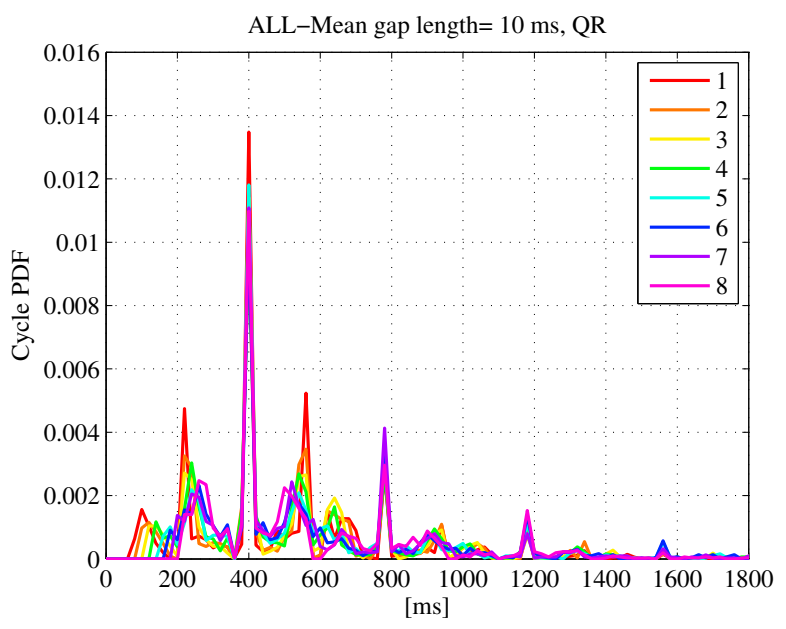

(c)

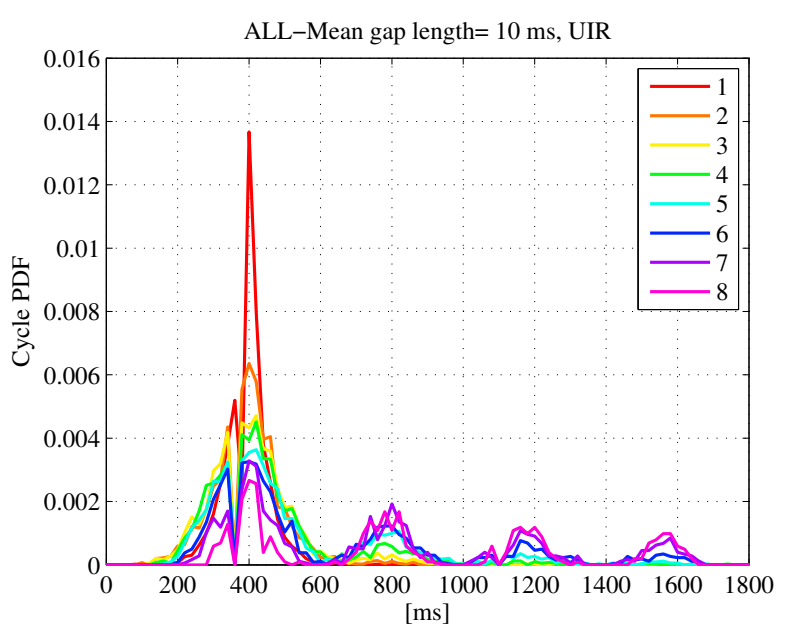

(b)

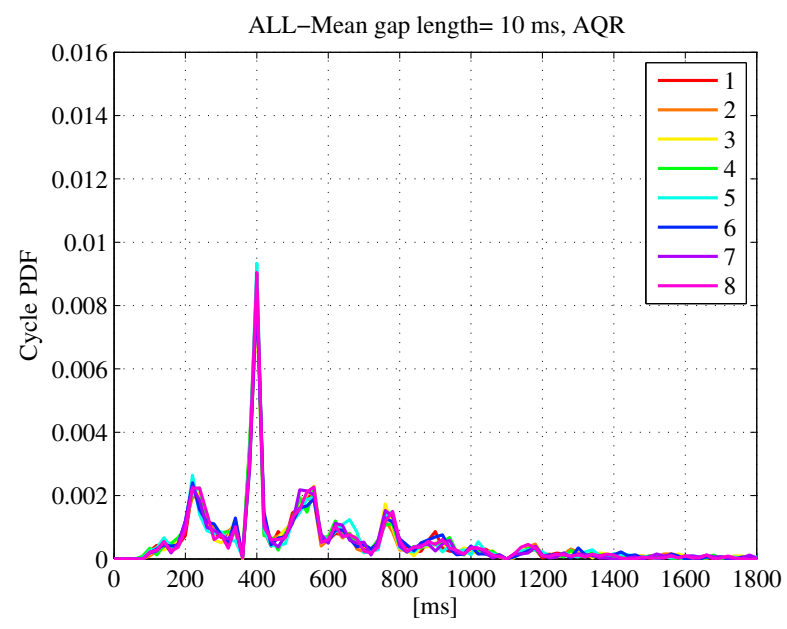

(d)

Fig. 12: Measured uplink cycle iat probability density function vs node ID (mean gap length $10 \mathrm{ms)} \mathrm{and} \mathrm{for:} \mathrm{(a)} \mathrm{BIR} \mathrm{policy;}$ (b) UIR policy; (c) QR policy; (d) AQR policy.

with the increase of the gap length. Observing Figure 9 we note that for gap length higher than $10 \mathrm{~ms}$, this scheme approaches the delivery rate of $\mathrm{AQR}$ and $\mathrm{QR}$, while the upper delay bound decreases. This however must be weighted with the unfairness of UIR scheme.

Summarizing, it can be said that the worst approach is BIR if we are interested in the packet delivery measure. Conversely, it shows the best behavior in term of "determinism" of the delivery, in the sense that if a transmission is successfully carried out we can also state what is the maximum delay for that transmission. This is a very interesting behavior in an industrial network context, especially if we are addressing realtime wireless networks.

The performances of $\mathrm{QR}$ and $\mathrm{AQR}$ are very similar, but AQR pays when analyzing fairness (confirming the results of Section V). UIR is a good solution in mean, but is the most unfair approach and probably the only one that could not be used in a real industrial system.

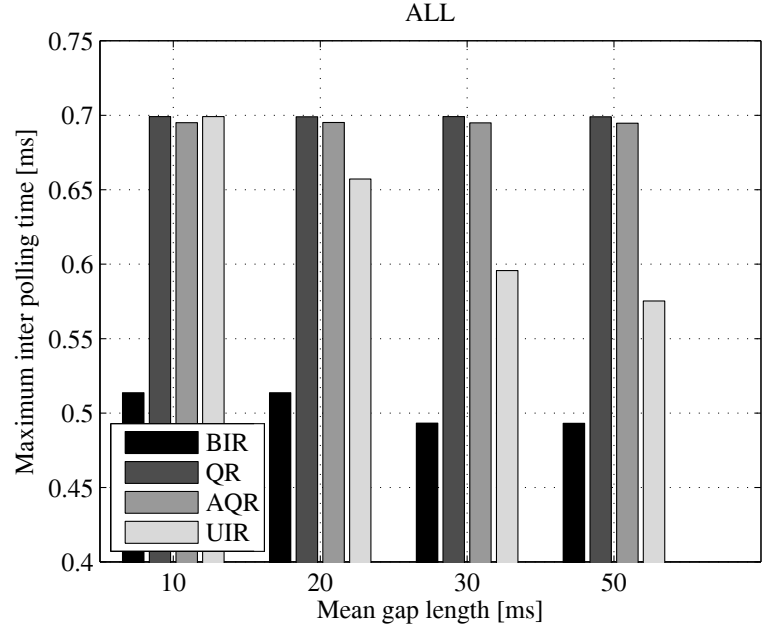

Fig. 13: Maximum inter polling time, taken among all the correct polling cycles. 


\section{CONCLUSIONS}

In this paper we have presented different retransmission strategies and investigated their performance (measured in the average fraction of unserved nodes per cycle and the fairness) under different interference scenarios, showing that the straightforward BIR scheme can be improved in different ways. By dropping the restriction on the number of retransmissions per node, by increasing the spacing between the first and second trial towards a node, or by adapting the poll sequence, the performance can be significantly improved. Especially the QR and AQR scheme offer a good balance between performance in terms of unserved nodes and fairness.

There is a lot of potential for future work. Firstly, it is worthwhile to consider more general interference patterns, for example incorporating real Wifi traffic. Secondly, it would be very interesting to incorporate transmit power control into the cyclic polling scheme. Thirdly, it is also interesting to investigate improvements of the simple-minded estimator used in this paper (see Equation 1), taking for example additional information about SNR and noise floor values into account.

\section{REFERENCES}

[1] G. Gamba, F. Tramarin, and A. Willig, "Retransmission strategies for cyclic polling over wireless channels in the presence of Interference," in Proc. 14th IEEE International Conference on Emerging Technologies and Factory Automation, ETFA 2009, Mallorca, Spain, Sept. 2009.

[2] J.-P. Thomesse, "Fieldbus Technology in Industrial Automation," Proceedings of the IEEE, vol. 93, no. 6, pp. 1073-1101, June 2005.

[3] A. Willig, "Recent and Emerging Topics in Wireless Industrial Communications: A Selection," IEEE Transactions on Industrial Informatics, vol. 4, no. 2, pp. 102-124, May 2008.

[4] A. Willig, K. Matheus, and A. Wolisz, "Wireless Technology in Industrial Networks," Proceedings of the IEEE, vol. 93, no. 6, pp. 1130-1151, June 2005 .

[5] Chipcon, $2.4 \mathrm{GHz}$ IEEE 802.15.4 / ZigBee-ready RF Transceiver, CC2420 Data Sheet, Chipcon Products from Texas Instruments, 2004.

[6] LAN/MAN Standards Committee of the IEEE Computer Society, IEEE Standard for Information technology - Telecommunications and information exchange between systems - Local and metropolitan area networks - Specific requirements - Part 15.4: Wireless Medium Access Control (MAC) and Physical Layer (PHY) Specifications for Low Rate Wireless Personal Area Networks (LR-WPANS), Sept. 2006, revision of 2006.

[7] I. Howitt, "WLAN and WPAN coexistence in UL band," Vehicular Technology, IEEE Transactions on, vol. 50, no. 4, pp. 1114-1124, Jul 2001.

[8] J. Howitt, I.; Gutierrez, "IEEE 802.15.4 low rate - wireless personal area network coexistence issues," Wireless Communications and Networking, 2003. WCNC 2003. 2003 IEEE, vol. 3, pp. 1481-1486 vol.3, 16-20 March 2003

[9] S. Y. Shin, H. S. Park, and W. H. Kwon, "Mutual interference analysis of IEEE 802.15.4 and IEEE 802.11b," Comput. Networks, vol. 51, no. 12, pp. 3338-3353, 2007.

[10] M. Bertocco, G. Gamba, A. Sona, and S. Vitturi, "Experimental characterization of wireless sensor networks for industrial applications," Instrumentation and Measurement, IEEE Transactions on, vol. 57, no. 8, pp. 1537-1546, Aug. 2008.

[11] M. Bertocco, G. Gamba, and A. Sona, "Is CSMA/CA really efficient against interference in a wireless control system? an experimental answer," Emerging Technologies and Factory Automation, 2008. ETFA 2008. IEEE International Conference on, pp. 885-892, Sept. 2008.

[12] T. Nandagopal and X. Gao, "Fair scheduling in wireless packet data networks," in Handbook of Wireless Networks and Mobile Computing, I. Stojmenovic, Ed. New York: John Wiley \& Sons, 2002, pp. 171-194.

[13] X. Wang, G. B. Giannakis, and A. G. Marques, "A Unified Approach to QoS-Guaranteed Scheduling for Channel-Adaptive Wireless Networks," Proceedings of the IEEE, vol. 95, no. 12, pp. 2410-2431, Dec. 2007.
[14] P. Bhagwat, P. Bhattacharya, A. Krishna, and S. K. Tripathi, "Using channel state dependent packet scheduling to improve TCP throughput over wireless LANs," Wireless Networks, vol. 3, no. 1, pp. 91-102, Mar. 1997.

[15] T. S. Rappaport, Wireless Communications - Principles and Practice. Upper Saddle River, NJ, USA: Prentice Hall, 2002.

[16] P. Graham, ANSI Common Lisp. Prentice Hall, 1995.

[17] K. Sohrabi, B. Manriquez, and G. J. Pottie, "Near ground wideband channel measurement in 800-1000 mhz," in Proc. IEEE Vehicular Technology Conference (VTC) '99. IEEE, 1999.

[18] A. F. Molisch, K. Balakrishnan, C.-C. Chong, S. Emami, A. Fort, J. Karedal, J. Kunisch, H. Schantz, U. Schuster, and K. Siwiak, "IEEE 802.15.4a channel model-final report," IEEE P802.15 Working Group for Wireless Personal Area Networks (WPANs), Tech. Rep. IEEE 802.1504-0662-01-04a, Sept. 2004.

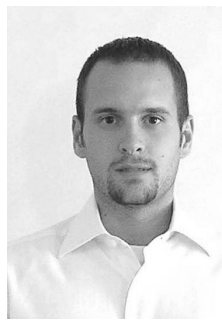

Giovanni Gamba (S '07) received the Laurea and Laurea Specialistica degrees (summa cum laude) in telecommunications engineering from the University of Padova, Padova, Italy, in 2004 and 2006, respectively. In 2007, he joined the Electronic Measurement Group, Department of Information Engineering, University of Padova. He obtained a Ph.D. degree in information technology at the Department of Information Engineering, University of Padova at the beginning of 2010. From January 2010 he is with the Institute of Electronics, Information Engineering and Telecommunications of the Italian National Council of Research, CNR-IEIIT. His main fields of interest are electromagnetic compatibility and wireless sensor networks. In particular, his works are tailored on interference study and mitigation between systems operating in the $2.4-\mathrm{GHz}$ band especially for industrial applications.

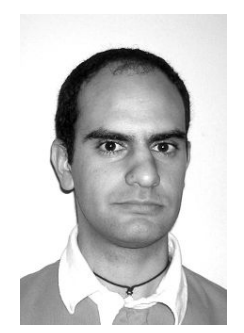

Federico Tramarin (S '07) is working toward the Ph.D. degree in Information Technology at the Department of Information Engineering at the University of Padova, Padova, Italy. He received the Laurea and Laurea Specialistica degrees (summa cum laude) in Electronic Engineering from the same University, in 2006 and 2008, respectively. In 2009, he joined the Electronic Measurement Group, Department of Information Engineering, University of Padova. His main fields of interest are electromagnetic compatibility and interference among different wireless sensor networks. In particular, his works are tailored on coexistence of industrial wireless systems operating in the $2.4-\mathrm{GHz}$ band and on electromagnetic compatibility issues on printed circuit boards.

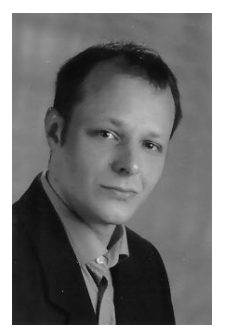

Andreas Willig (M) is a lecturer with the University of Canterbury, Christchurch since February 2010. Previously he was a senior researcher with the Telecommunication networks group (TKN) at the Technical University of Berlin, Germany since April 2005. From 2002 to 2005 he was an assistant professor with the Hasso-Plattner-Institue at University of Potsdam (Germany). He obtained the Dr.-Ing. degree in electrical engineering from Technical University Berlin (Germany) in 2002, and the diploma degree in computer science from University of Bremen (Germany) in 1994. His research interests include wireless networks, fieldbus and real-time systems, ad-hoc and sensor networks, all with specific focus on protocol design and performance aspects. 